\title{
Molecular characterisation of Sporothrix schenckii isolates from humans and cats involved in the sporotrichosis epidemic in Rio de Janeiro, Brazil
}

\author{
Rosani Santos Reis/ ${ }^{+}$, Rodrigo Almeida-Paes, Mauro de Medeiros Muniz, \\ Patrícia Morais e Silva Tavares, Paulo Cezar Fialho Monteiro, Tânia Maria Pacheco Schubach, \\ Maria Clara Gutierrez-Galhardo, Rosely Maria Zancopé-Oliveira
}

Laboratório de Micologia, Instituto de Pesquisa Clínica Evandro Chagas-Fiocruz, Av. Brasil 4365, 21045-900 Rio de Janeiro, RJ, Brasil

An epidemic of sporotrichosis, a subcutaneous mycosis caused by the fungus Sporothrix schenckii, is ongoing in Rio de Janeiro, Brazil, in which cases of human infection are related to exposure to cats. In an attempt to demonstrate the zoonotic character of this epidemic using molecular methodology, we characterised by DNA-based typing methods 19 human and 25 animal S. schenckii isolates from the epidemic, as well as two control strains. To analyse the isolates, the random amplified polymorphic DNA (RAPD) technique was performed using three different primers, together with DNA fingerprinting using the minisatellite derived from the wild-type phage M13 core-sequence. The analyses generated amplicons with considerable polymorphism. Although isolates exhibited high levels of genetic relatedness, they could be clustered into 5-10 genotypes. The RAPD profiles of epidemic $\mathrm{S}$. schenckii isolates could be distinguished from that of the United States isolate, displaying 20\% similarity to each primer and $60 \%$ when amplified with the M13 primer. DNA fingerprinting of S. schenckii isolated from the nails (42.8\%) and the oral cavities (66\%) of cats were identical to related human samples, suggesting that there is a common infection source for animals and humans in this epidemic. It is clear that cats act as a vehicle for dissemination of $\mathrm{S}$. schenckii.

Key words: Sporotrichosis - zoonotic transmission - RAPD - DNA fingerprinting

Sporotrichosis is a subcutaneous mycosis with a global distribution and an especial prevalence in Latin America and Africa (Rippon 1988, Diaz 1989, Kovarik et al. 2008, Schubach et al. 2008). Sporotrichosis is caused by the dimorphic fungus Sporothrix schenckii, which is associated with plant material and soil. This disease most often affects people with occupations related to soil handling (Diaz 1989) and it results from traumatic implantation of the agent into the skin (Rippon 1988).

The sporotrichosis outbreaks reported in the literature have been associated with contaminated plant material. The largest reported outbreak of sporotrichosis occurred in South Africa from 1941-1944, where around 3,000 miners were infected by wood props in the gold mines (Vismer \& Eicker 1994). Subsequently, an outbreak in the United States occurred in 1988 in which 84 individuals from 15 states were affected (Dixon et al. 1991). Hajjeh et al. (1997) reported an outbreak of sporotrichosis among tree nursery workers handling sphagnum moss. An outbreak in Australia involved individuals in contact with hay prior to infection (O'Reilly \& Altman 2006).

The random amplified polymorphic DNA (RAPD) method has been a useful tool in epidemiological investigations, especially for fungi, since prior knowledge

+ Corresponding author: rosani.reis@ipec.fiocruz.br

Received: 10 June 2008

Accepted: 30 June 2009 of the genome is not required. DNA techniques were used to examine clinical and environmental isolates of Sporothrix spp. from the 1988 sporotrichosis epidemic in the United States (Cooper et al. 1992). Recent molecular studies have assessed the genetic diversity of $S$. schenckii, demonstrating that different clusters of $S$. schenckii strains are related to different geographical origins (Marimon et al. 2006, O'Reilly \& Altman 2006, Gutierrez-Galhardo et al. 2008). Moreover, Marimon et al. (2007) proposed the existence of three new Sporothrix species through physiological and molecular studies.

Since 1998, there has been a sporotrichosis epidemic affecting humans, cats and in smaller proportions dogs in the metropolitan region of Rio de Janeiro, Brazil, mainly in areas with underprivileged socio-economic conditions and precarious health services. The most frequently affected individuals are homemakers taking care of cats with sporotrichosis (Barros et al. 2004). Epidemic isolates of $S$. schenckii from patients and cats have not been compared by molecular typing methods. In this study, the genetic polymorphisms of $S$. schenckii isolates from humans and associated cats from the sporotrichosis epidemic in state of Rio de Janeiro (RJ) were analysed using RAPD assays and PCR fingerprinting in an attempt to demonstrate the zoonotic character of the epidemic.

\section{PATIENTS, MATERIALS AND METHODS}

Sample description - Fourteen cases of cat-transmitted sporotrichosis in RJ were considered in this study (Table I). They occurred in four different municipal districts in RJ. These cases consisted of one or more cats with sporotrichosis and the humans that had contact 
with them. Eighteen humans and 16 cats were included. All humans related a history of contact with a sick cat before the onset of symptoms. Ten of the patients (55\%) also reported trauma by scratching ( 6 cases; $33 \%$ ) or biting (4 cases; $22 \%$ ) by sick cats on the sites of infection.

Fungi origin - We studied 44 S. schenckii strains, 19 from humans and 25 from cats (Table I). Two additional isolates from humans, one (17845) isolated in RJ, 1996 (before the occurrence of outbreaks), and one (24605) from the Dermatology Department of Columbia University, New York, were included in the analyses.

Fungal collection and identification - The isolates were obtained from clinical materials, as pus or biopsies of lesions from humans and felines and nails and swabs of oral cavities from felines. These samples were grown in Sabouraud dextrose agar and Mycobiotic slants (Becton, Dickinson and Company). Fungal identification was performed by conventional methods, which included morphology on PDA slants and microscopic observations of fungal fragments obtained after 15 days of incubation at RT. All isolates were tested for conversion to the yeast phase on BHI (brain heart infusion) agar at $37^{\circ} \mathrm{C}$ for seven days.

$D N A$ isolation - A single colony of yeast-phase $S$. schenckii was used to inoculate BHI broth, which was then grown at $37^{\circ} \mathrm{C}$ on a gyratory shaker at $120 \mathrm{rpm}$ for seven days to achieve sufficient cell mass for DNA extraction. Yeast cells were harvested and washed three times in sterile water. Genomic DNA was extracted and purified using the phenol-chloroform-isoamyl alcohol method (Woods et al. 1993). DNA quantification was done with a Gene Quant pro RNA/DNA Calculator (Amersham Pharmacia Biotech, Cambridge, UK).

$P C R-R A P D$ - For RAPD analysis, each strain was tested with three primers using Kit Ready-To-Go RAPD Analysis Beads (GE Healthcare). DNA amplification reactions were carried out in a $25 \mu \mathrm{L}$ volume containing $20 \mathrm{ng}$ of $S$. schenckii DNA and $25 \mathrm{pMol} / \mathrm{L}$ of primer. The other components were present on the beads. For amplification with primers 1 (5'-GGTGCGGGAA-3'), 4 (5'-AAGAGCCCGT-3') and 6 (5'-CCCGTCAGCA-3'), the thermal cycler (Peltier PTC200-MJ Research) was programmed for one denaturation cycle at $95^{\circ} \mathrm{C}$ for 5 min, followed by 45 cycles of denaturation at $95^{\circ} \mathrm{C}$ for 1 min, annealing at $36^{\circ} \mathrm{C}$ for $1 \mathrm{~min}$ and extension at $72^{\circ} \mathrm{C}$ for $2 \mathrm{~min}$, followed by a final extension period of $2 \mathrm{~min}$ at $72^{\circ} \mathrm{C}$. The randomly amplified DNA fragments were separated by electrophoresis on a $1 \%$ agarose gel in TBE buffer $(0.89 \mathrm{M}$ Tris, $0.89 \mathrm{M}$ boric acid, $0.02 \mathrm{M}$ disodium EDTA, $\mathrm{pH}$ 8.4) and stained with $0.5 \mu \mathrm{g} / \mathrm{mL}$ ethidium bromide (Roche Molecular Biochemicals). All visualised bands were counted and data were scored for the presence or absence of amplification products. Reproducibility was confirmed when identical profiles were observed in PCR assays repeated at least three times under the same conditions.

PCR fingerprinting - The minisatellite-specific core sequence of the wild-type phage M13 (5-'GAGGGTG-
GCGGTTCT-3') was used as a single primer in the PCR. The amplification reactions were performed as described by Gutierrez-Galhardo et al. (2008).

Computer-assisted data analysis - Similarity coefficients and Dice indices were determined for each isolate for the PCR fingerprinting and RAPD analyses using the NTSYSpc program (version 2.02h, Applied Biostatistic Inc). For clustering, the unweighted pair-group method with arithmetic means (UPGMA) was used.

\section{RESULTS}

DNA conditions and PCR standardisation - Extracted DNA analysed by agarose gel electrophoresis presented a single band without RNA or protein contamination. Six primers (Ready to Go Kit - RAPD analysis beads GE Healthcare - primers 1, 2, 3, 4, 5 and 6) were initially analysed to determine their discriminatory power. The primers 1, 4 and 6 were selected as they presented discriminatory indices of $0.79,0.87$ and 0.64 , respectively (data not shown).

RAPD analysis - Representative RAPD profiles obtained with all primers from the $46 \mathrm{~S}$. schenckii isolates indicated considerable polymorphism. Amplification reactions generated profiles composed of 8-15 bands ranging from 314-2357 bp in size. The genetic relationships obtained using the UPGMA method are represented as dendrograms (Fig. 1). The phylogenetic analysis identified 2-3 clonal groups, depending on the primer, which are highly related and contain contain 5-10 genotypes each. The combined data obtained using the three primers (primers 1, 4 and 6) clustered the $46 \mathrm{~S}$. schenckii isolates into three clonal groups exhibiting $90 \%$ relatedness, except for the US strain. RAPD patterns for the US isolate were completely different from the patterns observed among the other $S$. schenckii strains analysed by this methodology. The percentage of similarity was about $20 \%$ (data not shown).

M13 fingerprinting - The fingerprinting analysis revealed two major clusters containing the 46 strains, which were distinguished at a similarity distance of $80.8 \%$. The major cluster I (Fig. 2) included 39 strains clearly separated into two subgroups (Ia and Ib) with $90.8 \%$ similarity among the isolates. The isolates (75-1; 98-2 and $98-5$; $195-1$ and $195-2 ; 165-3$ and $165-5$ ) collected from cutaneous lesions and nails of four cats presented $100 \%$ similarity with the strains from their owners and sub-clustered into subgroup Ia together with the reference strain from RJ (17845). Subgroup Ib was composed of 10 isolates, six from cats and four from humans. This subgroup included isolates from a cutaneous lesion and oral cavity (721-4 and 721-1, respectively) of one cat with $100 \%$ relatedness to the strain (19182) isolated from its owner. Cluster II was composed of six S. schenckii isolates, exhibiting $85 \%$ relatedness among them. A clear separation into two subgroups (IIa, IIb) was observed within this cluster as well. Subgroup IIa accounted for two isolates from two different cats involved in case 3 (Table I) with $100 \%$ similarity between them and two human isolates from case 14 . Overall, the similarity be- 


\section{TABLE I}

Sporothrix schenckii strains isolated in the epidemic of sporotrichosis in state of Rio de Janeiro, Brazil, used in the study

\begin{tabular}{|c|c|c|c|c|}
\hline Case number & Strain number & Clinical specimen & Origin & Month/year \\
\hline \multirow[t]{3}{*}{1} & $15485-1$ & pus & human & $10 / 1998$ \\
\hline & $34-1$ & biopsy & cat & $10 / 1998$ \\
\hline & $34-3$ & nail & cat & $10 / 1998$ \\
\hline \multirow[t]{3}{*}{2} & $15647-1$ & pus & human & $12 / 1998$ \\
\hline & 47 & nail & cat & 03/1999 \\
\hline & 48 & biopsy & cat & 03/1999 \\
\hline \multirow[t]{5}{*}{3} & $15677-1$ & pus & human & $01 / 1999$ \\
\hline & $15677-4$ & pus & human & $01 / 1999$ \\
\hline & $41-1$ & nail & cat a & $02 / 1999$ \\
\hline & $41-2$ & biopsy & cat a & 02/1999 \\
\hline & $51-1$ & biopsy & cat b & 05/1999 \\
\hline \multirow[t]{2}{*}{4} & $16393-2$ & pus & human & 07/1999 \\
\hline & $97-2$ & pus & cat & 08/1999 \\
\hline \multirow[t]{2}{*}{5} & 16415 & biopsy & human & 07/1999 \\
\hline & $99-2$ & biopsy & cat & 08/1999 \\
\hline \multirow[t]{2}{*}{6} & 16459 & biopsy & human & 07/1999 \\
\hline & $75-1$ & biopsy & cat & 08/1999 \\
\hline \multirow[t]{3}{*}{7} & 16672 & pus & human & 09/1999 \\
\hline & $142-1$ & nail & cat & 09/1999 \\
\hline & $142-2$ & biopsy & cat & 09/1999 \\
\hline \multirow[t]{4}{*}{8} & 17878 & pus & human & 08/1999 \\
\hline & $98-2$ & biopsy & cat a & 08/1999 \\
\hline & $98-5$ & nail & cat a & 08/1999 \\
\hline & 335 & oral swab & cat $\mathrm{b}^{a}$ & $05 / 2000$ \\
\hline \multirow[t]{3}{*}{9} & $16910-1$ & pus & human & $11 / 1999$ \\
\hline & $195-1$ & biopsy & cat & $11 / 1999$ \\
\hline & $195-2$ & nail & cat & $11 / 1999$ \\
\hline \multirow[t]{4}{*}{10} & 17500 & biopsy & human & $04 / 2000$ \\
\hline & $165-1$ & oral swab & cat & $10 / 1999$ \\
\hline & $165-3$ & biopsy & cat & $10 / 1999$ \\
\hline & $165-5$ & nail & cat & 10/1999 \\
\hline \multirow[t]{3}{*}{11} & 19182 & pus & human & $05 / 2001$ \\
\hline & $721-1$ & oral swab & cat & $05 / 2001$ \\
\hline & $721-4$ & pus & cat & $05 / 2001$ \\
\hline \multirow[t]{2}{*}{12} & 19481 & biopsy & human & $07 / 2001$ \\
\hline & $792-1$ & pus & cat & $06 / 2001$ \\
\hline \multirow[t]{5}{*}{13} & 19536 & pus & human & $07 / 2001$ \\
\hline & 19540 & biopsy & human & 07/2001 \\
\hline & 19541 & biopsy & human & $07 / 2001$ \\
\hline & 19549 & pus & human & $07 / 2001$ \\
\hline & 856 & blood culture & cat & $07 / 2001$ \\
\hline \multirow[t]{3}{*}{14} & 19777 & pus & human & $09 / 2001$ \\
\hline & 19778 & pus & human & $09 / 2001$ \\
\hline & 975 & pus & cat & $09 / 2001$ \\
\hline $\operatorname{Ref}^{b}$ & 17845 & pus & human & 1996 \\
\hline Ref. $^{b}$ & 24605 & unknown & human & unknown \\
\hline
\end{tabular}

$a$ : asymptomatic; $b$ : the 17845 and 24605 samples were used as reference strains. 
A

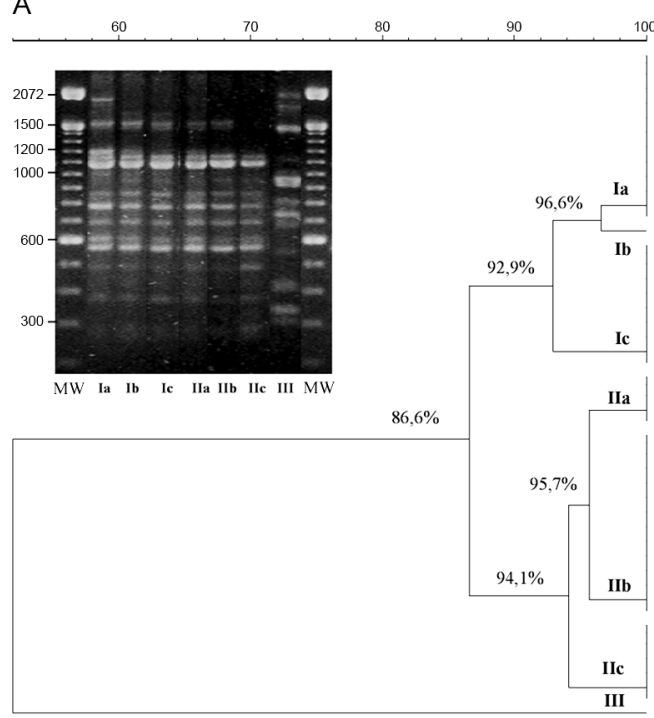

B

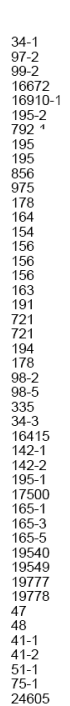

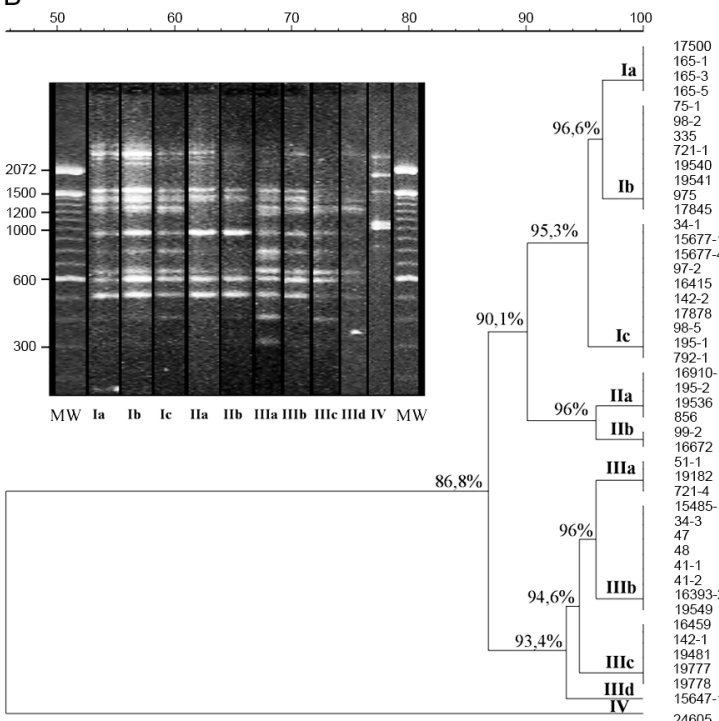

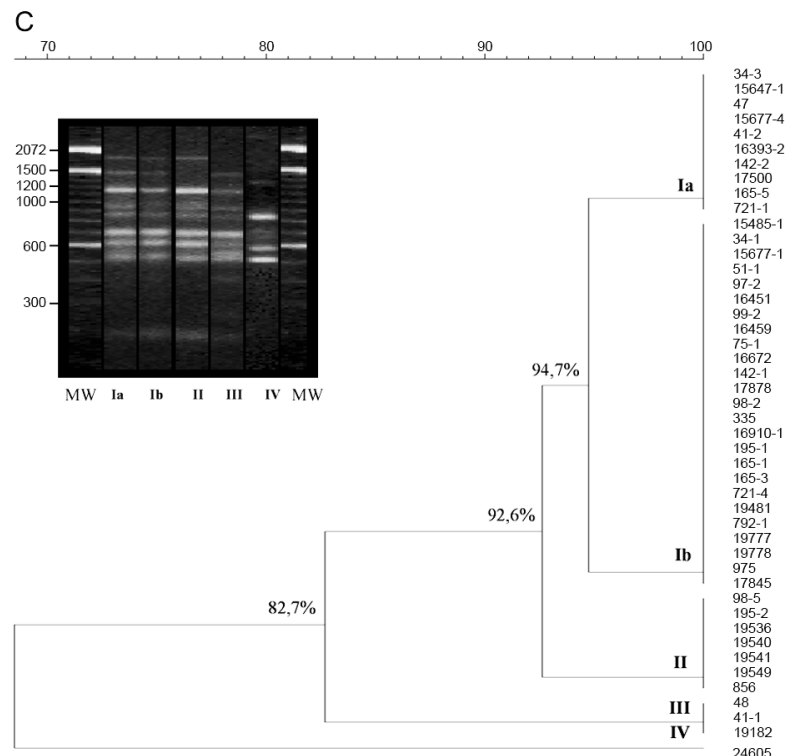

Fig. 1: representative profiles and dendrograms obtained by DNA analyses of 44 Sporothrix schenckii strains involved on the epidemic of sporotrichosis in Rio de Janeiro, Brazil, and two control strains by RAPD using primer 1 (A), primer 4 (B) and primer 6 (C).

tween the US strain and the strains from RJ was about $64 \%$. It is interesting to note that all samples from cases $6,8,9,10,11$ and 13 (Table I) presented the same DNA fingerprinting when analysed with primer M13.

Correlation between cat and human strains - Total identities between $S$. schenckii isolates from lesions, nails and oral cavities of cats are listed in Table II with their respective primers. It is notable that when the primers 1 and 6 for the RAPD technique and M13 for DNA fingerprinting were used, the same percentage (42.8\%) of similarity was shown among the $S$. schenckii isolated from the nails of the cats and the isolates from their owners. With RAPD primer 1, the three strains obtained from the oral cavities of cats were identical to the related patients' isolates. However, with primers 4 and 6 , just one of them
(165-1 and 335, respectively) presented an identical genotype to the patient's isolate. Two of these isolates (165-1 and 721-1) were identical based on PCR fingerprinting.

\section{DISCUSSION}

Molecular epidemiology is based mainly on the analysis of chromosomal polymorphisms. Information on the genetic diversity of $S$. schenckii isolates associated with the sporotrichosis epidemic would be very useful to public health authorities in designing and implementing prevention and intervention strategies. The DNA typing, using the RAPD assay and PCR fingerprinting of $46 S$. schenckii isolates, was carried out in an initial effort to identify molecular types from humans and associated cats affected by the epidemic in RJ. The RAPD tech- 


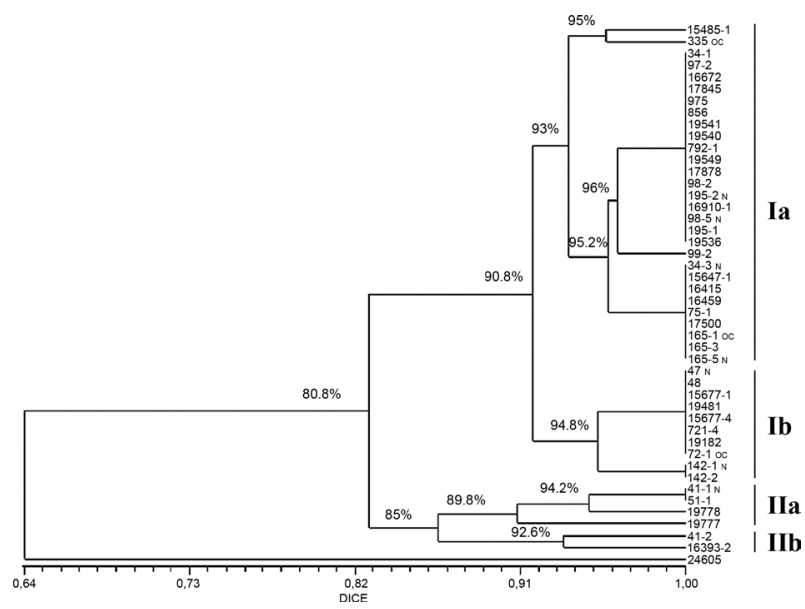

Fig. 2: DNA fingerprinting of two controls and 44 epidemic Sporothrix schenckii strains using minisatellite derived from the wild-type phage M13 core-sequence.

\section{TABLE II}

Percentage of identical genotypic profile between Sporothrix schenckii isolates from cats and those from their owners

\begin{tabular}{lcccc}
\hline & $\begin{array}{c}\text { Primer 1 } \\
\%\end{array}$ & $\begin{array}{c}\text { Primer 4 } \\
\%\end{array}$ & $\begin{array}{c}\text { Primer 6 } \\
\%\end{array}$ & $\begin{array}{c}\text { M13 } \\
\%\end{array}$ \\
\hline Skin lesion $(\mathrm{n}=13)$ & 23 & 15.3 & 61.5 & 38.5 \\
Nail $(\mathrm{n}=7)$ & 42.8 & 57.1 & 42.8 & 42.8 \\
Oral lesion $(\mathrm{n}=3)$ & 100 & 33.3 & 33.3 & 66.6 \\
\hline
\end{tabular}

nique and DNA fingerprinting using the M13 primer were chosen for this study since they are easy and rapid genotyping methods and they have become quite popular for fungal analysis.

Recently, we demonstrated that 59 human epidemic strains were genetically related through molecular typing with DNA fingerprinting and ITS sequence analysis. These results suggest that the strains isolated from the sporotrichosis epidemic originated from a common source (GutierrezGalhardo et al. 2008). Our data corroborate this hypothesis and confirm that cats play a major role in the spread of sporotrichosis in RJ since the $S$. schenckii isolates from cats are closely related to those isolated from humans.

The DNA profiles of $S$. schenckii exhibited considerable similarity and did not cluster together according to their geographical area. No variation in the genetic patterns of S. schenckii isolates obtained between 19982001 in this RJ epidemic was observed. Furthermore, the genetic profile of the reference strain (17845) collected in 1996, before the beginning of the epidemic, was similar to the others. The high similarity among these isolates suggests the possibility that only one virulent genetic population is present in the epidemic environments and that one or more populations of S. schenckii are maintained in nature for long periods of time.
Our group previously reported the isolation of $S$. schenckii from the nails of cats with sporotrichosis that infected their owners by scratching. We suggested that the vehicle of transmission could be the cat's nails (Schubach et al. 2001). In this study, we verified this hypothesis by molecular methods, showing between $42.8-57.1 \%$ total identity between samples from cat's nails and humans.

Three $S$. schenckii isolates from the oral cavity of cats were included in this study. One of them (335 strain) was isolated from an asymptomatic cat that had contact with other cats with sporothrichosis in the same house. In this case (case 8), strain 98-2 was isolated from a symptomatic cat of the same household that died nine months before the isolation of strain 335. Both strains (98-2 and 335) presented the same genotypic profile. Strain 335 presented $95.3-100 \%$ similarity with the patient strain (strain 17878), suggesting that just one genotype is involved in this case and indicating that $S$. schenckii can persist inside the domiciliary area for prolonged periods of time. Two other isolates from oral cavities were obtained from cats with restricted cutaneous lesions. In case 10 we observed $94.7-100 \%$ similarity between the strain from the oral cavity of the cat (165-1) and the lesion of the human patient (17500), indicating this patient acquired S. schenckii from its cat. Our data are the first to demonstrate this zoonotic potential through molecular analysis.

In $23-61.5 \%$ of our cases, isolates from cats' skin lesions and from the associated humans were identical, depending on the primer used in the PCR reactions. Reed et al. (1993) have reported one case of zoonotic transmission of sporotrichosis as determined by genotypic analysis of the isolates from both the human and the cat involved. The RFLP profiles of the strains from this single outbreak were identical. Our data corroborate the above considerations about the high zoonotic potential of domestic cats in sporotrichosis.

Some patients $(45 \%)$ included in this work did not report any kind of trauma, but they developed sporotrichosis when they were taking care of their sick animals. The strains isolated from humans and the sick cats showed high genetic relatedness. S. schenckii strains from cases 6 and 10 were identical based on DNA fingerprinting, emphasising again the zoonotic potential of the disease. The infected cats present a high S. schenckii burden in their lesions (Reed et al. 1993, Schubach et al. 2004) and can thus act as a source of infection to the human if there is a contact between a pre-existent wound in the patient and the exudates from cat's lesions.

At present, no teleomorph is known for $S$. schenckii (de Beer et al. 2003); however, we found, as with previous studies (Mesa-Arango et al. 2002, O'Reilly \& Altman 2006, Gutierrez-Galhardo et al. 2008), some genetic variability in $S$. schenckii, especially when analysing strains from different countries. The band pattern of the US strain was totally different from those of Brazil, which is in accordance with results from other studies of S. schenckii, as well as with other pathogenic fungi, such as Paracoccidioides brasiliensis and Histoplasma capsulatum (Muniz et al. 2001, Mesa-Arango et al. 2002, Hahn et al. 2003). However, the genetic variability or 
polymorphic patterns found in different geographical areas can also be due to different ecological processes or genetic factors that promote these polymorphisms (Mesa-Arango et al. 2002). Moreover, we cannot discard the possibility that new Sporothrix species are associated with the RJ epidemics, as suggested by Marimon et al. (2007). In addition, the US isolate included in this study most probably represents the real $S$. schenckii in the new species complex. Studies are in progress in our laboratory to corroborate these previously reported data.

In conclusion, the RAPD technique is an efficient tool for the investigation of the relationship between $S$. schenckii isolated from human subjects and felines and can be used to distinguish among 5-10 strain profiles included in this study. A similar result was obtained when the DNA samples were amplified using primer M13. Our results show a strong correlation between the isolates from humans and their respective cats in RJ and support the idea that contact with cats infected by $S$. schenckii constitutes a route of sporotrichosis infection.

\section{ACKNOWLEDGEMENTS}

To Andreia Pussenti Derossi and Celuta Sales Alviano, for providing the control strains used in this study.

\section{REFERENCES}

Barros MBL, Schubach AO, Valle ACF, Gutierrez-Galhardo MC, Conceição-Silva F, Schubach TMP, Reis RS, Wanke B, Marzochi KBF, Conceição MJ 2004. Cat-transmitted sporotrichosis epidemic in Rio de Janeiro, Brazil: description of a series of cases. Clin Infect Dis 38: 529-535.

Cooper CR, Breslin BJ, Dixon DM, Salkin IF 1992. DNA typing of isolates associated with the 1988 sporotrichosis epidemic. J Clin Microbiol 30: 1631-1635.

de Beer ZW, Harrington TC, Vismer HF, Wingfield BD, Wingfield MJ 2003. Phylogeny of the Ophiostoma stenoceras - Sporothrix schenckii complex. Mycologia 95: 434-441.

Diaz IAC 1989. Epidemiology of sporotrichosis in Latin America. Mycopathologia 103: 113-116.

Dixon DM, Salkin IF, Hurd NJ, Haines JH, Kemna ME, Coles FB 1991. Isolation and characterization of Sporothrix schenckii from clinical and environmental sources associated with the largest US epidemic of sporotrichosis. J Clin Microbiol 29: 1106-1113.

Gutierrez-Galhardo MC, Zancopé-Oliveira RM, Valle AC, AlmeidaPaes R, Tavares PMS, Monzon A, Mellado E, Rodriguez-Tudela JL, Cuenca-Estrella M 2008. Molecular epidemiology and antifungal susceptibility patterns of Sporothrix schenckii isolates from a cat-transmitted epidemic of sporotrichosis in Rio de Janeiro, Brazil. Med Mycol 46: 141-151.

Hahn RC, Macedo AM, Fontes CJF, Batista RD, Santos NL, Hamdan JS 2003. Random amplified polymorphic DNA as a valuable tool for epidemiological studies of Paracoccidioides brasiliensis. J Clin Microbiol 41: 2849-2854.

Hajjeh R, McDonnell S, Reef S, Licitra C, Hankins M, Toth B, Padhye A, Kaufman L, Passarell L, Cooper C, Hutwagner L, Hopkins R, McNiel M 1997. Outbreak of sporotrichosis among tree nursery workers. J Infect Dis 176: 499-504.

Kovarik CL, Neyra E, Bustamante B 2008. Evaluation of cats as the source of endemic sporotrichosis in Peru. Med Mycol 46: 53-56.

Marimon R, Cano J, Gene J, Sutton DA, Kawasaki M, Guarro J 2007. Sporothrix brasiliensis, $S$. globosa and $S$. mexicana, three new Sporothrix species of clinical interest. J Clin Microbiol 45: 31983206.

Marimon R, Gene J, Cano J, Trilles L, Dos Santos Lazera M, Guarro J 2006. Molecular phylogeny of Sporothrix schenckii. J Clin Microbiol 44: 3251-3256.

Mesa-Arango AC, Reyes-Montes MR, Pérez-Mejía A, Navarro-Barranco H, Souza V, Zúñiga G, Toriello C 2002. Phenotyping and genotyping of Sporothrix schenckii isolates according to geographic origin and clinical form of sporotrichosis. J Clin Microbiol 40: 3004-3011.

Muniz MM, Pizzini CV, Peralta JM, Reiss E, Zancopé-Oliveira RM 2001. Genetic diversity of Histoplasma capsulatum strains isolated from soil, animals and clinical specimens in Rio de Janeiro State, Brazil, by PCR-based random amplified polymorphic DNA assay. J Clin Microbiol 39: 4487-4497.

O'Reilly LC, Altman SA 2006. Macrorestriction analysis of clinical and environmental isolates of Sporothrix schenckii. J Clin Microbiol 44: 2547-2552.

Reed KD, Moore FM, Geiger GE 1993. Zoonotic transmission of sporotrichosis: case report and review. Clin Infect Dis 16: 384387.

Rippon JW 1988. Sporotrichosis. In JW Rippon, Medical mycology, WB Saunders, Philadelphia, p. 325-352.

Schubach A, Barros MBL, Wanke B 2008. Epidemic sporotrichosis. Curr Opin Infect Dis 21: 129-133.

Schubach TMP, Schubach AO, Okamoto T, Barros MBL, Figueiredo FB, Cuzzi T, Monteiro PCF, Reis RS, Perez MA, Wanke B 2004. Evaluation of an epidemic of sporotrichosis in cats: 347 cases (1988-2001). J Am Vet Med Assoc 224: 1623-1629.

Schubach TMP, Valle ACF, Gutierrez-Galhardo MC, Monteiro PCF, Reis RS, Zancopé-Oliveira RM, Marzochi KBF, Schubach A 2001. Isolation of Sporothrix schenckii from the nails of domestic cats (Felis catus). Med Mycol 39: 147-149.

Vismer HF, Eicker A 1994. Growth of human pathogenic isolates of Sporothrix schenckii on indigenous and exotic wood species in South Africa. Mycol Res 98: 121-124.

Woods JP, Kersulyte D, Goldman WE, Berg DE 1993. Fast DNA isolation from Histoplasma capsulatum: methodology for arbitrary primer polymerase chain reaction-based epidemiological and clinical studies. J Clin Microbiol 31: 463-464. 